\title{
Need for technologies in advanced corneal research, diagnosis, and transplantation
}

\section{Neil Lagali}

Neil Lagali, "Need for technologies in advanced corneal research, diagnosis, and transplantation," Proc. SPIE 10858, Ophthalmic Technologies XXIX, 108580B (28 February 2019); doi: 10.1117/12.2520656 


\title{
Need for technologies in advanced corneal research, diagnosis, and transplantation.
}

\author{
Neil Lagali \\ Department of Ophthalmology, Institute for Clinical and Experimental Medicine, Linköping \\ University, Linköping, SE-581 83, Sweden
}

\begin{abstract}
Never before have optical, information, and biomedical technologies converged to the extent they do today. Moreover, the accessibility of enormous amounts of computing power at reasonable cost has transformed raw data into useable information that can enable biological discoveries and medical decisions to be made faster and more accurate than ever before. In the field of ophthalmology in particular, advanced optical instrumentation, devices, and procedures have revolutionized the standard of care and improved outcomes for millions, for the benefit of society. The cornea is the clear outer window of the eye and is directly accessible for examination and treatment using light-based approaches, and as such it provides us with a unique window into the physiology of the body in health and disease. At the same time, the cornea is a model tissue from which we have acquired much knowledge about light-tissue interactions. Finally, and importantly, diseases of the cornea compromising its transparency are responsible for millions of cases of corneal blindness globally, so there is much to gain from technological advancements in the field. Here, a need for new technological solutions is presented, that is not primarily technology-driven but instead motivated by real and pressing medical needs in the research, diagnosis and treatment of corneal blindness.
\end{abstract}

Keywords: corneal blindness, transplantation, ophthalmology, photodynamic therapy, confocal microscopy, keratitis, tear film, tissue engineering

\section{CORNEAL BLINDNESS - A GLOBAL BURDEN}

According to the World Health Organization (WHO), corneal blindness is the fourth largest cause of blindness globally accounting for $5.1 \%$ of blindness ${ }^{1}$. While this alone represents over 10 million cases, the number of people with visual impairment due to corneal disease, trauma, and pathologies is much higher. The burden is moreover unequally distributed, with the low-to-middle income countries having the most cases of corneal pathologies and at the same time the poorest access to adequate treatment ${ }^{2}$. Once the cornea becomes scarred, the loss of transparency is irreversible and traditionally a donated human cadaver cornea is used as a tissue source for transplantation to the damaged or diseased cornea. This traditional approach, however, depends upon the availability, procurement, storage, and timely distribution of suitable donor corneas. Unfortunately, this tissue procurement and eye banking infrastructure is lacking in those areas of the world in most need, resulting in millions of cases of preventable corneal blindness and vision impairment.

\section{TISSUE ENGINEERING THE CORNEA AND FEMTOSECOND LASER SURGERY}

To address the high global demand for corneas - a biological transparent optical element - research has been undertaken to develop a man-made alternative to the human cornea that is suitable for transplantation ${ }^{3}$. One approach that has thus far been the most successful in terms of long-term implantation in humans, is the collagen-based hydrogel ${ }^{4,5}$, although another approach using de-cellularized porcine corneas is currently under initial clinical investigation ${ }^{6}$. These technologies suggest that the natural protein collagen, of which the human cornea is primarily composed, may be an ideal raw material from which to engineer a manmade cornea. Although still in an early development phase, these technologies indicate that engineering the cornea could be a viable approach, in particular when combined with innovative implantation techniques using a femtosecond laser, to optimize wound healing ${ }^{7}$. The femtosecond laser enables precision cutting of the cornea owing to the cornea's natural transparency and thus the ability to focus the light to a given depth within the tissue. This results in the possibility of performing complex surgical procedures such as extraction of diseased or damaged tissue, from within the cornea without significantly disrupting the surface epithelial

Ophthalmic Technologies XXIX, edited by Fabrice Manns, Per G. Söderberg, Arthur Ho, Proc. of SPIE Vol. 10858, 108580B · C 2019 SPIE · CCC code: 1605-7422/19/\$18 · doi: 10.1117/12.2520656 
cells, thereby improving postsurgical wound healing ${ }^{7}$. Although the femtosecond laser is used in ophthalmology surgery today, we are still only at a nascent stage in achieving its full potential. More sophisticated hardware and software could be used, for example, to scan the cornea in three dimensions (using OCT) to record the exact areas of opacities (occurring for example due to scars or dystrophies). This information could then be used to guide the femtosecond laser to precisely remove the affected region(s) while leaving the surrounding tissue intact. In a related application, precise shaping of the cornea could be performed from within the corneal tissue (leaving the surface intact) by using the femtosecond laser to vaporize small regions of tissue to correct refractive errors. This would be an improvement over today's laser refractive surgery which cuts nerves and disrupts the corneal epithelium leading to protracted wound healing ${ }^{8}$. Uncorrected refractive errors resulting in visual impairment represent a collective group of hundreds of millions worldwide according to the $\mathrm{WHO}^{9}$.

The tissue engineered cornea is also in a very early stage of technological development, but limited clinical results suggest that the insertion of this inert optical element into the cornea is a feasible treatment for certain types of corneal blindness ${ }^{4,5}$. Moreover, initial results with newer collagen-based materials indicate that the engineered collagen-based cornea is not completely inert but rather permits invasion of host cells and subsequent degradation of the implanted collagen ${ }^{7}$. Still, such an implant is considered a passive device. The next generation of implants may be more 'active', meaning that in addition to restoring transparency to the cornea, they perform other functions such as the release of drugs or other biological factors ${ }^{10}$. Slow or sustained-release delivery of drugs or biologics (growth factors, recombinant proteins or antibodies) into the cornea via an implant would be a major improvement over transplantation followed by manual eye drop administration. There is additionally an untapped need to monitor drug release or implanted material breakdown or integration into the recipient cornea, and this could be achieved through optical methods. For example, a hydrogel can be manufactured to be transparent in a central optical zone, while having increased optical density in the periphery, such that this peripheral zone is visible after implantation into the cornea. Over time, as the hydrogel integrates into the recipient through breakdown and replacement by new collagen, the optical density in the peripheral zone will change and this can be monitored by direct imaging ${ }^{11}$. This is just one example of optical monitoring; as drug delivery into the eye becomes more sophisticated, optical technologies can play an important role in monitoring the presence and distribution of drugs or other factors in the tissue. This could be achieved, for example, by chemically linking the substance with a naturally fluorescent compound or by exploiting intrinsic optical absorption of drugs or other compounds in specific wavelength regions. In follow-up visits, for example, the physician would simply illuminate the cornea with light of a certain wavelength or a spectral camera could be used to image optical transmission/absorption, in order to determine presence or efficacy of a drug or pathogen.

\section{PHOTODYNAMIC THERAPY AND CORNEAL CROSSLINKING}

The therapeutic potential of light goes beyond laser-based cutting or ablation of corneal tissue, and includes photodynamic therapy, of which the UVA/riboflavin collagen crosslinking (CXL) treatment is a prime example. In CXL, the goal is to strengthen or stiffen the corneal collagen, to prevent its weakening and degradation in diseases such as keratoconus. In this procedure the corneal epithelium is typically removed to improve penetration of a riboflavin (vitamin B2) solution, which is applied to the eye's surface. After the cornea is soaked with riboflavin, it is exposed to a specific wavelength and fluence of UVA light, which through the action of riboflavin as a photosensitizer, creates extra cross-links between collagen fiber bundles, thereby stiffening the cornea and increasing its resistance to degradation or weakening ${ }^{12}$. In recent years, the CXL technique has evolved to treat a broader range of pathologies including corneal infectious keratitis, as an initial obliteration of all cellular material in the irradiated zone could theoretically also destroy harmful pathogens, thereby eradicating them and preventing their spread. This cell death is due to the reactive oxygen species generation that disrupts microbial and cellular membranes. This approach of CXL is termed PACK-CXL (photoactivated chromophore for infectious keratitis CXL) to distinguish its use from the goal of mechanically strengthening the cornea ${ }^{13}$. PACK-CXL has been used to successfully treat cases of bacterial, fungal, and parasitic infection of the cornea; however, re-treatments are sometimes necessary and outcomes are not always optimal ${ }^{13}$. While the dose or fluence of the UVA light has been varied to give a more or less aggressive effect, the CXL procedure relies on UVA light and riboflavin as a sensitizer. 
Other sensitizers also exist, but have not yet been fully explored or applied in human corneas. For example, toluidine blue $\mathrm{O}$ is a photosensitizer activated by red light $(633 \mathrm{~nm})$, and has been shown in vitro to eradicate periodontal pathogens ${ }^{14}$ and is well-studied in the field of dentistry. Toluidine blue eye drops are used clinically as a vital stain to detect cancerous changes in the ocular surface ${ }^{15}$, but have not yet been tested in combination with red light in the cornea. In a similar vein, in a recent in vitro study, the Actanthamoeba castellani parasite (in its trophozoite and cyst forms) was exposed to Rose Bengal, a dye commonly used in ophthalmology to detect poor protection of the ocular surface epithelium, that can lead to ocular surface dryness. Rose Bengal was found to be a photosensitizer when green light of wavelength $523 \mathrm{~nm}$ was used, and at a total irradiation dose of $5.4 \mathrm{~J} / \mathrm{cm}^{2}$ the Acanthamoeba were eradicated in vitro ${ }^{16}$. This remains to be tested in vivo but has been successful against fungal infections in patients ${ }^{17}$ holds promise for treatment of acanthamoeba keratitis, a serious infection that is difficult to treat and can lead to blindness ${ }^{18}$. What other combinations of vital dyes and light could have therapeutic benefits against difficult-to-treat corneal conditions?

'Classical' photodynamic therapy using verteporfin as a photosensitizer and exposure to $689 \mathrm{~nm}$ red light has been used to reduce the number and patency of abnormal vessels from the cornea to restore its transparency ${ }^{19}$. More recently, even the CXL procedure has been demonstrated in an animal model to induce apoptosis of vascular endothelial cells thereby regressing pathologic corneal vessels and improving transparency ${ }^{20}$. A major benefit of these approaches is the targeting of unwanted cells and microbes by light, without affecting the surrounding collagen of the cornea. While normal corneal cells such as epithelium and stromal keratocytes also undergo apoptosis during these procedures, the epithelium naturally regenerates while keratocytes repopulate the stroma over time. Even more selective photosensitizing agents could in the future avoid the need to kill healthy cells, and only target the pathogenic entities.

\section{OPTICAL DIAGNOSTIC TECHNOLOGIES IN THE CORNEA}

Optical diagnostic technologies such as optical coherence tomography/angiography, in vivo confocal microscopy, specular microscopy, scheimpflug photography, meibomography, etc. are breakthroughs allowing us to observe, study and diagnose disease with high precision and efficacy. These technologies enable us to image the cornea in detail in new ways. While the instrumentation undoubtedly enables new ways of observing and thinking about disease, there is often a missing link in terms of understanding how the new imaging modalities relate to healthy and diseased tissue and how they can be used to detect and diagnose abnormalities and disease. It is often left to biomedical researchers and clinicians to determine the best ways to use and interpret the data. There is a large untapped need for software-based expert systems that can analyze large databases of images to determine image correlations with diseases, in a more accurate and consistent manner than any human observer could. One example is the 'deep learning' method, which has been applied to classify age-related macular degeneration and diabetic macular edema from a database of over 200,000 retinal OCT images, with sensitivity and specificity over $98 \%{ }^{21}$.

Deep learning and other machine-based image segmentation, tracing, calculation and classification methods are required in many areas of ophthalmology, particularly in the cornea. For example, the computerized assessment of Meibomian glands by meibomography has recently been used to develop an objective grading scale for Meibomian gland assessment in patients ${ }^{22}$. For certain conditions such as acanthamoeba keratitis, however, such a computer-assisted grading or detection does not exist today. Instead, images of acanthamoeba cysts obtained clinically by in vivo confocal microscopy (IVCM) are assessed subjectively by either experienced or unexperienced observers, which can radically affect the diagnostic result by up to a factor of two in sensitivity and specificity of diagnosis ${ }^{23}$. Here, a machine-based learning system could be trained to recognize specific image features indicative of parasitic trophozoites and/or cysts that correlate with a positive diagnosis, to improve the diagnosis based on IVCM images. Because this condition can seriously and rapidly impair vision, quick diagnosis and proper treatment is paramount and much could be gained from applying machine-based techniques here.

IVCM, which is a live, non-invasive laser-scanning confocal microscope using red laser light to scan the corneal layers, has also been used to non-invasively image corneal nerves in health and disease ${ }^{24}$. The corneal nerves are small-fiber nerves that are part of the peripheral nervous system, and have been shown to parallel the nerve degeneration in diseases

such as diabetes mellitus ${ }^{25}$, Parkinson's disease ${ }^{26}$, and multiple sclerosis ${ }^{27}$. Thus, assessing corneal nerves non-invasively could provide a sensitive means to assess systemic neurodegeneration, before clinical symptoms appear. Moreover, imaging of nerves in the cornea in two or three dimensions provides more information than the thin tissue sections 
obtained by invasive skin biopsy. The imaging and quantification of corneal nerves however, is largely subjective and this has led to discrepancies in reported parameters in the literature ${ }^{25}$. Automated detection, segmentation, and tracing of corneal nerves in IVCM images is required, and has been applied in some studies to determine normal baseline characteristics $^{28,29}$, however, a single efficient algorithm should be made openly available. Likewise, image feature detection algorithms could be used to automate the recognition and detection of different subsets of dendritic cells from IVCM images, which have been shown to be elevated with the onset of diabetes and in multiple sclerosis ${ }^{27,30}$. Deep learning techniques may also have a great potential here, for assessment of corneal nerve plexi to detect nerve patterns indicative of the onset and/or progression of specific neurodegenerative diseases. This is best achieved through wide-area

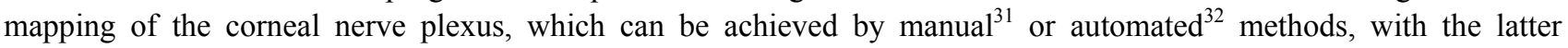
providing higher quality images in a fraction of the time required for manual assembly.

\section{TEAR FILM DIAGNOSTICS - A NEW FRONTIER?}

It is becoming increasingly evident that there is a close interplay between proteins in the tear film and the cornea, and the ability to non-invasively sample and measure the tears provides new opportunities for understanding and diagnosing disease. Analysis of tear proteins by multiplex or proteomic methods allows precision quantification of growth factors, with tear cytokine profiling gaining much popularity for the study of corneal disease. Profiles of multiple cytokines have been shown to be linked with various corneal and ocular disease including glaucoma, dry eye, and aniridia ${ }^{33-35}$. Optical properties of the tear film are likely also perturbed with disease, although these have yet to be measured in this context; properties such as optical absorption/transmission, scatter, and birefringence could be measured in vivo or in tear samples ex vivo. Tear cytokine profiles may also affect the spectral properties of the tears in a measurable way. Rapid, point-ofcare diagnostics with light would greatly simplify tear film analysis. Besides conventional spectroscopy, Raman spectroscopy could be used to probe tear chemical composition in a rapid manner. Specific chemical dyes or other compounds could be made to react with tear samples, to give characteristic optical signatures indicative of disease. The 'tear ferning test' is an alternative approach where a tear sample is dried on a microscope slide and analyzed, with the crystallization pattern of the tear residues being indicative of the tear status ${ }^{36}$. This test has not been widely used but could be further combined with optical techniques. The advantage of using the tear film is its accessibility, ease of collection, its transparency, its complex protein content mirroring the status of the eye (due to soluble factors secreted from corneal cells), and its potential for rapid eye diagnostics.

\section{CONCLUSIONS}

Optical technologies have revolutionized the field of ophthalmology, for the benefit of millions. Still, hundreds of millions are visually impaired or blind. Much preventable blindness can be addressed by the development of advanced technologies and devices such as through tissue engineering and optical surgery, which although in their infancy today could in the future become much more sophisticated and reach broader populations. Conversely, many areas where existing technologies are utilized today could benefit from more automated and intelligent systems to sift through large amounts of data to improve our analysis, understanding and decision-making abilities. Technology alone is not enough; intelligent systems interpreting the outputs in terms of real human disease are required. The potential for light as a therapeutic and diagnostic tool is just starting to be realized, although many opportunities for better, more effective and more targeted treatments and diagnostics exist. Future development of light-based technologies should acknowledge practical problems in ophthalmology clinics affecting patient care, include the software and algorithmic component, and be tailored to a specific disease and/or clinical question of significance.

\section{REFERENCES}

[1] https://www.who.int/blindness/causes/priority/en/index8.html

[2] Gain, P., Jullienne, R., He, Z., Aldossary, M., Acquart, S., Cognasse, F., and Thuret, G., "Global survey of corneal transplantation and eye banking," JAMA Ophthalmol 134(2), 167-173 (2016). 
[3] Matthyssen, S., Van den Bogerd, B., Dhubhghaill, S.N., Koppen, C., and Zakaria, N., "Corneal regeneration: A review of stromal replacements," Acta Biomater (2018) Feb 1.

[4] Fagerholm, P., Lagali, N.S., Merrett, K., Jackson, W.B., Munger, R. et al., "A biosynthetic alternative to human donor tissue for inducing corneal regeneration: 24-month follow-up of a phase 1 clinical study," Sci Transl. Med 2(46), 46ra61 (2010).

[5] Fagerholm, P., Lagali, N.S., Ong, J.A., Merrett, K., Jackson, W.B. et al., "Stable corneal regeneration four years after implantation of a cell-free recombinant human collagen scaffold," Biomaterials 35(8), 2420-2427 (2014).

[6] ClinicalTrials.gov website, Url: https://clinicaltrials.gov/ct2/show/NCT03105466 . "Prospective study of deep anterior lamellar keratoplasty using acellular porcine cornea."

[7] Koulikovska, M., Rafat, M., Petrovski, G., Veréb, Z., Akhtar, S., Fagerholm, P., and Lagali, N. "Enhanced regeneration of corneal tissue via a bioengineered collagen construct implanted by a nondisruptive surgical technique,” Tissue Eng. Pt. A 21(5-6), 1116-1130 (2015).

[8] Erie, J.C., McLaren, J.W., Hodge, D.O., and Bourne, W.M., "Recovery of corneal subbasal nerve density after PRK and LASIK,” Am. J. Ophthalmol 140(6), 1059-64 (2005).

[9] https://www.who.int/blindness/causes/priority/en/index4.html

[10] Ali, Z., Islam, A., Sherrell, P., Le-Moine. M., Lolas, G. et al., "Adjustable delivery of pro-angiogenic FGF-2 by collagen-alginate microspheres," Biology Open (2018) Jan 1:bio-027060.

[11] Rafat, M., Xeroudaki, M., Koulikovska, M., Sherrell, P., Groth, F., Fagerholm, P., and Lagali, N., "Composite coreand-skirt collagen hydrogels with differential degradation for corneal therapeutic applications," Biomaterials 83(3), 142-55 (2016).

[12] Randleman, J.B., Khandelwal, S.S., and Hafezi, F., "Corneal cross-linking," Surv. Ophthalmol 60(6), 509-23 (2015).

[13] Tabibian, D., Mazzotta, C., and Hafezi, F., "PACK-CXL: corneal cross-linking in infectious keratitis," Eye and Vision 3(1),11 (2016).

[14] Nielsen, H.K., Garcia, J., Væth, M., and Schlafer, S., "Comparison of riboflavin and toluidine blue O as photosensitizers for photoactivated disinfection on endodontic and periodontal pathogens in vitro," PloS One 10(10), e0140720 (2015).

[15] Romero, I.L., de Nadai Barros, J., Martins, M.C., and Ballalai, P.L., "The use of 1\% toluidine blue eye drops in the diagnosis of ocular surface squamous neoplasia," Cornea 32(1), 36-39 (2013).

[16] Atalay, H.T., Dogruman-Al, F., Sarzhanov, F., Özmen, M.C., Tefon, A.B., Arıbaş, Y.K., and Bilgihan, K., "Effect of Riboflavin/Rose Bengal-Mediated PACK-CXL on Acanthamoeba Trophozoites and Cysts in Vitro," Curr. Eye Res 43(11), 1322-1325 (2018).

[17] Amescua, G., Arboleda, A., Nikpoor, N., Durkee, H., Relhan, N., et al., "Rose Bengal photodynamic antimicrobial therapy: a novel treatment for resistant Fusarium keratitis," Cornea 36(9), 1141-1144 (2017).

[18] Lorenzo-Morales, J., Khan, N.A., and Walochnik, J., "An update on Acanthamoeba keratitis: diagnosis, pathogenesis and treatment," Parasite 22, 10 (2015).

[19] Al-Torbak, A.A., "Photodynamic therapy with verteporfin for corneal neovascularization," Mid. East Afr. J. Ophthalmol 19(2), 185 (2012).

[20]Hou, Y., Le, V.N., Tóth, G., Siebelmann, S., Horstmann, J., et al., "UV light crosslinking regresses mature corneal blood and lymphatic vessels and promotes subsequent high risk corneal transplant survival," Am. J. Transplant (2018) Apr 19.

[21] Li, F., Chen, H., Liu, Z. et al., "Fully automated detection of retinal disorders by image-based deep learning," Graefes Arch Clin Exp Ophthalmol (2019). https://doi.org/10.1007/s00417-018-04224-8.

[22] Adil, M.Y., Xiao, J., Olafsson, J., Chen, X., Lagali, N.S., et al., "Meibomian gland morphology is a sensitive early indicator of Meibomian gland dysfunction," Am. J. Ophthalmol (2018) Dec 19. pii: S0002-9394(18)30675-5. doi: 10.1016/j.ajo.2018.12.006.

[23] Hau, S.C., Dart, J.K., Vesaluoma, M., Parmar, D.N., Claerhout, I., et al., "Diagnostic accuracy of microbial keratitis with in vivo scanning laser confocal microscopy,” Br. J. Ophthalmol 94, 982-987 (2010). 
[24] Cruzat, A., Qazi, Y., and Hamrah, P., "In vivo confocal microscopy of corneal nerves in health and disease," Ocul Surf. 15(1), 15-47 (2016).

[25]De Clerck, E.E., Schouten, J.S., Berendschot, T.T., Kessels, A.G., Nuijts, R.M., et al., "New ophthalmologic imaging techniques for detection and monitoring of neurodegenerative changes in diabetes: a systematic review," Lancet Diab. Endocrinol 3(8), 653-663 (2015).

[26] Kass-Iliyya, L., Javed, S., Gosal, D., Kobylecki, C., Marshall, A., et al., "Small fiber neuropathy in Parkinson's disease: a clinical, pathological and corneal confocal microscopy study," Parkinsonism Rel. Disorders 21(12), 14541460 (2015).

[27]Bitirgen, G., Akpinar, Z., Malik, R.A., and Ozkagnici, A. "Use of corneal confocal microscopy to detect corneal nerve loss and increased dendritic cells in patients with multiple sclerosis," JAMA Ophthalmol 135(7), 777-782 (2017).

[28] Parissi, M., Karanis, G., Randjelovic, S., Germundsson, J., Poletti, E., Ruggeri, A., Utheim, T.P., and Lagali, N., "Standardized baseline human corneal subbasal nerve density for clinical investigations with laser-scanning in vivo confocal microscopy," Invest. Ophthalmol. Vis. Sci 54(10), 7091-7102 (2013).

[29] Tavakoli, M., Ferdousi, M., Petropoulos, I.N., Morris, J., Pritchard, N., et al., "Normative values for corneal nerve morphology assessed using corneal confocal microscopy: a multinational normative data set," Diabetes Care Jan 29:dc142311 (2015).

[30] Lagali, N.S., Badian, R.A., Liu, X., Feldreich, T.R., et al., "Dendritic cell maturation in the corneal epithelium with onset of type 2 diabetes is associated with tumor necrosis factor receptor superfamily member 9," Sci Reports 8(1), 14248 (2018).

[31] Patel, D.V., and McGhee, C.N., "Mapping of the normal human corneal sub-basal nerve plexus by in vivo laser scanning confocal microscopy," Invest. Ophthalmol. Vis. Sci 46(12), 4485-4488 (2005).

[32] Lagali, N.S., Allgeier, S., Guimaraes, P., Badian, R.A., Ruggeri, A., et al., "Reduced corneal nerve fiber density in type 2 diabetes by wide-area mosaic analysis," Invest. Ophthalmol. Vis. Sci 58(14), 6318-6327 (2017).

[33] Gupta, D., Wen, J.C., Huebner, J.L., Stinnett, S., Kraus, V.B., et al., "Cytokine biomarkers in tear film for primary open-angle glaucoma," Clin. Ophthalmol 11, 411 (2017).

[34] Na, K.S., Mok, J.W., Kim, J.Y., Rho, C.R., and Joo, C.K., "Correlations between tear cytokines, chemokines, and soluble receptors and clinical severity of dry eye disease," Invest. Ophthalmol. Vis. Sci 53(9), 5443-5450 (2012).

[35] Landsend, E.C., Utheim, Ø.A., Pedersen, H.R., Aass, H.C., Lagali, N., et al., "The level of inflammatory tear cytokines is elevated in congenital aniridia and associated with Meibomian gland dysfunction," Invest. Ophthalmol. Vis. Sci 59(5), 2197-2204 (2018).

[36] Masmali, A.M., Purslow, C., and Murphy, P.J., "The tear ferning test: a simple clinical technique to evaluate the ocular tear film," Clin. Exp. Optom 97(5), 399-406 (2014). 